\title{
Cerebral Hyperperfusion Syndrome and Related Conditions
}

\author{
Montserrat González Delgado ${ }^{a} \quad$ Julien Bogousslavsky ${ }^{b}$ \\ ${ }^{a}$ Neurology Department, Central University Hospital of Asturias, Oviedo, Spain; \\ ${ }^{b}$ Neurology Department, Clinique Valmont, Montreux, Switzerland
}

\section{Keywords}

Cerebral hyperperfusion syndrome $\cdot$ Hypertensive encephalopathy - Posterior reversible encephalopathy syndrome $\cdot$ Reversible cerebral vasoconstriction syndrome

\begin{abstract}
Cerebral vasoconstriction is a normal physiological response under determined conditions to preserve a normal cerebral blood flow. However, there are several syndromes, with impaired cerebral autoregulation and cerebral vasoconstriction, not related with infection or inflammation, which share the same radiological and clinical presentation. We review here the cerebral hyperperfusion syndrome and related conditions such as hypertensive encephalopathy, posterior reversible encephalopathy syndrome, and reversible cerebral vasoconstriction syndrome. These syndromes might share the same pathophysiological mechanism with endothelial damage, cerebral vasoconstriction, blood-brain barrier disturbance, cerebral edema, and, occasionally, intracerebral hemorrhage, with fatal cases described in all. Despite knowledge of these syndromes, they still remain unknown to us. Why these entities present in some patients and not in others goes further than the actual understanding of these diseases. We have to consider that a genetic susceptibility and molecular disturbances may be involved. Thus, more studies are needed in order to better characterize such syndromes.
\end{abstract}

(c) 2020 S. Karger AG, Basel

\section{Introduction}

Cerebral vasoconstriction is a normal physiological response under determined conditions to preserve a normal cerebral blood flow (CBF). However, there are several syndromes, with impaired cerebral autoregulation and cerebral vasoconstriction, which are not related with infection or inflammation but share the same pathophysiological mechanism and clinical presentation. We review here the cerebral hyperperfusion syndrome (CHS) and related conditions, such as hypertensive encephalopathy (HE), posterior reversible encephalopathy syndrome (PRES), and reversible cerebral vasoconstriction syndrome (RCVS).

\section{Cerebral Autoregulation}

Anterior cerebral arterial circulation can be divided into 2 types of arteries: the conducting vessels, consisting of the internal carotid artery and its major intracerebral trunks, and the penetrating arterioles, which arise from the previous ones and enter the brain parenchyma. Under normal conditions, there is only a $10-15 \%$ decrease in perfusion pressure between the origin of the internal carotid artery and the penetrating vessels [1]. Approximately $80 \%$ of cerebrovascular resistance is related to the small arteries, arterioles, and capillary bed, with the remaining $20 \%$ contributed by postcapillary venules and veins. 
Cerebral autoregulation is managed by the main resistance vessels, the arterioles, which have a myogenic and a neurogenic component [2]. Due to the myogenic component, increased intravascular pressure results in vasoconstriction of small arterioles. When blood pressure exceeds the limit of the myogenic component, autoregulation in large arterioles and small arteries is dependent on sympathetic autonomic innervation [3]. Cerebral vessels possess a rich sympathetic neural supply, and sympathetic stimulation will increase the upper limit of autoregulation [2]. As a result of sparse sympathetic innervation, the vertebrobasilar system is less protected than the anterior circulation [3].

The capacity for autoregulatory vasoconstriction is much smaller (8-10\% of baseline diameter) than that for autoregulatory vasodilation (up to $65 \%$ of baseline diameter) [4]. Therefore, much greater changes in cerebral blood volume occur with hypotension than with hypertension [4].

\section{Cerebral Ischemia-Reperfusion Injury}

With a mean arterial pressure above $150-160 \mathrm{~mm} \mathrm{Hg}$, animal studies demonstrate a breakthrough phenomenon, with passive arteriolar dilation, pinocytotic fluid transfer, injury to the capillary bed, vasogenic edema, and vessel injury with altered arterial morphology [2]. With a mean arterial pressure of $200 \mathrm{~mm} \mathrm{Hg}$, vessel morphological changes tend to be permanent [2]. Several factors can alter the upper tolerated blood pressure limit, namely, sympathetic stimulation or chronic hypertension [2]. Above the upper arterial pressure limits, the vessels lose their ability to autoregulate vascular resistance in response to changes in blood pressure, and dysautoregulation appears $[5,6]$.

Described as normal perfusion pressure breakthrough theory, Spetzler et al. [7] showed a patient diagnosed as having ischemic symptoms secondary to a steal phenomenon from an arteriovenous malformation (AVM). The side of the AVM resulted in a chronically ischemic and chronically dilated arterial network; when normal perfusion was suddenly reestablished after the resection of the AVM, the pressure led to capillary breakthrough, resulting in edema and hemorrhage [7].

Similarly, the failure of cerebral autoregulation at high pressures is also the presumed mechanism after carotid revascularization where sudden augmentation of blood flow and pressure may surpass this control system [8]. The resulting cellular damage in a previously viable is- chemic tissue is defined as ischemia-reperfusion injury [8]. This is characterized by oxidant production, complement activation, and increased microvascular permeability, resulting in an impaired blood-brain barrier (BBB), which could lead to intracerebral edema, early and delayed cell death, and intracranial hemorrhage following reperfusion [8].

\section{Cerebral Hyperperfusion Syndrome}

CHS was initially described with carotid endarterectomy (CEA) by Sundt et al. [1], but it may present in both CEA and carotid artery stenting. Patients may present headaches, visual disturbances, seizures, neurological deficit, and confusional episodes.

The pathophysiological mechanism of CHS remains only partially understood. The chronic low-flow state induced by severe carotid disease results in a compensatory dilation of cerebral vessels distal to the stenosis, as part of the normal autoregulatory response, to maintain adequate $\mathrm{CBF}[5,6]$. In this chronically dilated state, the vessels lose their ability to autoregulate vascular resistance in response to changes in blood pressure [5, 6]. In fact, it has been shown that this dysautoregulation is proportional to the duration and severity of chronic hypoperfusion [9]. After revascularization and reperfusion, the impaired cerebral autoregulation could then contribute to a cascade of intracranial microcirculatory changes [10], as explained above, with an inability of reaction toward the augmentation of the $\mathrm{CBF}$ after the carotid recanalization. $\mathrm{CBF}$ after such an intervention typically falls by the seventh postoperative day, but autoregulation may take up to 6 weeks to improve [9].

Anatomopathological study of a patient with a CHS showed, in small arteries and arterioles throughout the affected hemisphere, reactive changes consisting of the swelling of endothelial cells and hyperplasia of such and other blood vessel cells, in addition to fibrinoid necrosis and a marked edema of the adjacent brain [11]. These features are similar to the changes seen in cases of malignant hypertension [11].

However, CHS can already occur in the presence of moderate hyperperfusion [12]. Because of this, some authors have suggested that the hemodynamic pathogenetic mechanisms of the CHS are more complicated than previously believed and that they may be more accurately described by the term "reperfusion syndrome" [12]. 
Fig. 1. CHS, HE, PRES, and RCVS shared the same pathophysiological mechanism, with an overlapping of clinical and radiological findings. Luxury perfusion syndrome would be related with an initial stage of CHS. CHS, cerebral hyperperfusion syndrome; HE, hypertensive encephalopathy; PRES, posterior reversible encephalopathy syndrome; RCVS, reversible cerebral vasoconstriction syndrome.

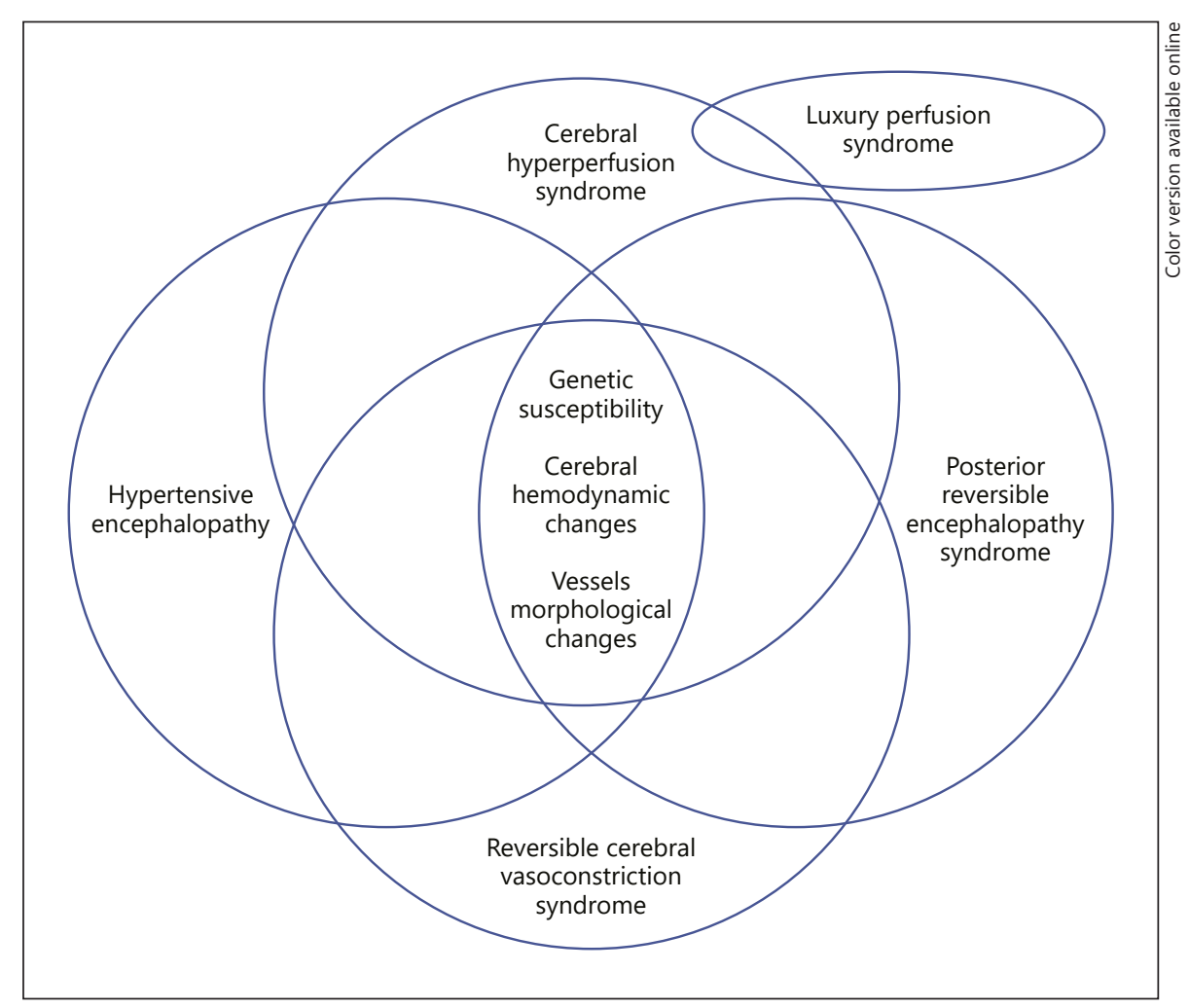

\section{CHS and Related Conditions}

Some conditions may share the same pathophysiological mechanism of CHS, with radiological and clinical similarities, an overlapping being seen among them (shown in Fig. 1). In this sense, an interrelation between CHS and HE [1, 10, 11], HE and PRES [13], and PRES and RCVS has been described [14-16]. We support the possibility of an interrelation among all of them, and between the luxury perfusion syndrome and CHS.

\section{Luxury Perfusion Syndrome}

In 1966, Lassen described a reactive hyperemia after hypoxia as the luxury perfusion syndrome [17]. When normal flow is reestablished, the increase in CBF is approximately $20-40 \%$, but a luxury perfusion may occur due to failure of autoregulation in the dilated vessels [18]. That is, even a few minutes of hypoxia is followed by reactive hyperemia lasting hours, and probably such hyperemia lasts much longer than would be needed to reoxygenate the brain [17]. Since the vessels are already dilated at a normal pressure, cerebral autoregulation is expected to be diminished or absent in this state of luxury perfusion [17]. As an increased permeability of the BBB is a characteristic finding after hypoxia, a rise in systemic arterial blood pressure might well aggravate the disease by development of local edema and hemorrhage [17]. Classical CHS would be regarded as a serious manifestation on the clinical spectrum of reperfusion injury [19].

\section{Hypertensive Encephalopathy}

Oppenheimer et al. [20] coined in 1928 the term HE as acute episodes of cerebral phenomena (epileptiform convulsions, coma, headache, amaurosis, hemiplegia, and aphasia) as the manifestation of circulatory disturbances in the brain as a consequence of hypertension. At that time, an anatomopathological study of these patients showed that cerebral vasoconstriction and cerebral edema were present in this syndrome [20].

In 1984, Bernstein et al. [11] presented a patient with the clinical triad of unilateral head, face, and eye pain; seizures; and delayed intracerebral hemorrhage after CEA. The anatomopathological findings of this case suggested that the microcirculation had become ill-adapted to with- 
stand normal perfusion pressure and had reacted to such in a manner analogous to that exhibited by normal vessels when exposed to sudden elevated perfusion pressure as in malignant hypertension [11]. This was interpreted as an acute $\mathrm{HE}$ in a hemisphere rendered susceptible by the loss of autoregulation [21].

Thus, the normal perfusion pressure breakthrough in an ischemic cortex is similar to the hypertensive breakthrough in the normal cortex [7]. In both situations, the regulating capacity of the vessels is surpassed [7].

Some authors have previously hypothesized a close relationship between CHS and HE as both entities shared some features clinically, pathologically, and radiographically [10]. The principal abnormalities in CHS and HE include white matter edema, predominantly affecting the posterior cerebral circulation due to scarce sympathetic innervation; both may potentially suffer hemorrhagic or ischemic infarction [10].

\section{Posterior Reversible Encephalopathy Syndrome}

Described in 1996 by Hinchey et al. [22], the PRES is an acute and progressive syndrome associated with predominantly white matter edema in the parietal-temporal and occipital regions related to sympathetic innervations $[2,23]$. Later descriptions have shown PRES in other cerebral regions such the frontal lobe or brainstem [15]. Patients may present with headaches, visual disturbances, altered consciousness, or seizures. Controversy exists over the hyper- or hypoperfusion mechanism of PRES, and it is possible that both mechanisms play a role in PRES and CHS.

Hypertension could provoke a loss of autoregulation and subsequent hyperperfusion, resulting in endothelial damage, dilatation of cerebral arterioles, and disruption of the BBB [2]. Despite the fact that the relation of PRES and hypertension is well known, severe hypertension is not mandatory and may develop at much lower blood pressure levels [23], such as CHS.

Alternatively, endothelial dysfunction and vasoconstriction may provoke a cerebral hypoperfusion which may lead to altered integrity of the BBB in PRES, developing brain edema [23]. Evidence of acute and chronic vessel injuries has been described in autopsy studies as intimal thickening, segmental vessel narrowing, intimal dissection, and organized thrombi [23]. Neuroimaging studies in these patients, including conventional angiography, have shown blood vessel irregularities consistent with vasoconstriction $[15,23]$. This endothelial injury may be seen in malignant hypertension [16]. As in the syndromes described above, PRES may be not reversible in some cases, and permanent neurological deficit may occur as a result of hemorrhagic or ischemic infarction $[2,24]$.

\section{Reversible Cerebral Vasoconstriction Syndrome}

RCVSs were described by Calabrese et al. [24] as being characterized by severe headaches, with or without other acute neurological symptoms (such as transient or persistent focal deficits and seizures). Transient hypertension, which can be marked, is not uncommon [24].

The characteristic angiographic findings in RCVS are alternating areas of arterial constriction and dilatation, often called beading, in multiple vascular beds [24]. These findings are similar to those of PRES [25]. Alternating areas of constriction and normal vascular caliber, rather than areas of dilatation, can also be seen [24]. Most specific evidence for RCVS is the reversibility of vasoconstriction within 3 months [24]. A vasogenic edema that usually completely reverses in a few days may occur in RCVS, but cytotoxic edema, cerebral infarction, or hemorrhage may occur [24, 25].

Some authors have suggested an interrelation between PRES and RCVS [14-16]. Like PRES, 2 hypotheses (hyper-and hypoperfusion) have been suggested [14]. Severe arterial hypertension leads to an autoregulation failure with hyperperfusion and edema [14]. Alternatively, endothelial dysfunction could have a role in both disorders, suggesting a gene dysfunction, such as polymorphism in the gene encoding BDNF, which has previously been implicated in both sympathetic overactivity and endothelial dysfunction [25].

\section{Conclusion}

CHS, HE, PRES, and RCVS might share the same pathophysiological mechanism with cerebral vasoconstriction, BBB disturbance, cerebral edema, and occasionally, intracerebral hemorrhage, with fatal cases being described in all of them. Despite knowledge of these syndromes, they still remain little understood by us. Why these entities present in some patients and not in others goes further than the actual understanding of these diseases. We have to consider that genetic susceptibility and molecular disturbances may be involved. Thus, more studies are needed in order to better characterize such syndromes. 


\section{Conflict of Interest Statement}

The authors have no conflicts of interest to declare.

\section{Author Contributions}

M.G.D. was responsible for the literature search and discussion. J.B. revised it critically for important intellectual content and approved the final version.

\section{Funding Sources}

The authors did not receive any funding.

\section{References}

1 Sundt TM Jr, Sharbrough FW, Piepgras DG, Kearns TP, Messick JM Jr, O’Fallon WM. Correlation of cerebral blood flow and electroencephalographic changes during carotid endarterectomy: with results of surgery and hemodynamics of cerebral ischemia. Mayo Clin Proc. 1981;56(9):533-43.

2 Bartynski WS. Posterior reversible encephalopathy syndrome. Part 2: controversies surrounding pathophysioloty of vasogenic edema. Am J Neurorad. 2008;29:1043-9.

3 van Mook WN, Rennenberg RJ, Schurink GW, van Oostenbrugge RJ, Mess WH, Hofman PA, et al. Cerebral hyperperfusion syndrome. Lancet Neurol. 2005;4(12):877-88.

4 Rangel-Castilla L, Spetzler RF, Nakaji P. Normal perfusion pressure breakthrough theory: a reappraisal after 35 years. Neurosurg Rev. 2015;38(3):399-5.

5 Abou-Chebl A, Yadav JS, Reginelli JP, Bajzer C, Bhatt D, Krieger DW. Intracranial hemorrhage and hyperperfusion syndrome following carotid artery stenting: risk factors, prevention, and treatment. J Am Coll Cardiol. 2004;43(9):1596-601.

6 Coutts SB, Hill MD, Hu WY. Hyperperfusion syndrome: toward a stricter definition. Neurosurgery. 2003;53(5):1053-60.

7 Spetzler RF, Wilson CB, Weinstein P, Mehdorn M, Townsend J, Telles D. Normal perfusion pressure breakthrough theory. Clin Neurosurg. 1978;25:651-72.

8 Adhiyaman V, Alexander S. Cerebral hyperperfusion syndrome following carotid endarterectomy. QJM. 2007;100(4):239-44.
9 Rafiq MK, Connolly D, Randall M, Blank C. Cerebral hyperperfusion syndrome. Pract Neurol. 2014;14(1):64-6.

10 Naylor AR, Evans J, Thompson MM, London NJ, Abbott RJ, Cherryman G, et al. Seizures after carotid endarterectomy: hyperperfusion, dysautoregulation or hypertensive encephalopathy? Eur J Vasc Endovasc Surg. 2003;26(1):39-44.

11 Bernstein M, Fleming JF, Deck JH. Cerebral hyperperfusion after carotid endarterectomy: a cause of cerebral hemorrhage. Neurosurgery. 1984;15(1):50-6.

12 Karapanayiotides T, Meuli R, Devuyst G Piechowski-Jozwiak B, Dewarrat A, Ruchat P, et al. Postcarotid endarterectomy hyperperfusion or reperfusion syndrome. Stroke. 2005; 36(1):21-6.

13 Miller JB, Suchdev K, Jayaprakash N, Hrabec D, Sood A, Sharma S, et al. New developments in hypertensive encephalopathy. Curr Hypertens Rep. 2018;20(2):1-7.

14 Ducros A, Bousser MG. Reversible cerebral vasoconstriction syndrome. Pract Neurol. 2009;9(5):256-67.

15 Fugate JE, Rabinstein AA. Posterior reversible encephalopathy syndrome: clinical and radiological manifestations, pathophysiology, and outstanding questions. Lancet Neurol. 2015;14(9):914-25.

16 Levitt A, Zampolin R, Burrns J, Bello JA, Slasky SE. Posterior reversible encephalopathy syndrome and reversible cerebral vasoconstriction syndrome. Distinct clinical entities with overlapping pathophysiology. Radiol Clin North Am. 2019;57(6):1133-46.
17 Lassen NA. The luxury-perfusion syndrome and its possible relation to acute metabolic acidosis localised within the brain. Lancet. 1966;2(7473):1113-5.

18 Russell DA, Gough MJ. Intracerebral haemorrhage following carotid endarterectomy. Eur J Vasc Endovasc Surg. 2004;28(2):11523.

19 Cho AH, Cho YP, Lee DH, Kwon TW, Kwon SU, Suh DC, et al. Reperfusion injury on magnetic resonance imaging after carotid revascularization. Stroke. 2014;45(2):602-4.

20 Oppenheimer BS, Fishbery AM. Hypertensive encephalopathy. Arch Intern Med. 1928; 41(2):264-78.

21 Moulakakis KG, Mylonas SN, Sfyroeras GS, Andrikopoulos V. Hyperperfusion syndrome after carotid revascularization. J Vasc Surg. 2009;49(4):1060-8

22 Hinchey J, Chaves C, Appignani B, Breen J, Pao L, Wang A, et al. A reversible posterior leukoencephalopathy syndrome. N Engl J Med. 1996;334(8):494-500.

23 Ay H, Buonanno FS, Schaefer PW, Le DA, Wang B, Gonzalez RG, et al. Posterior leukoencephalopathy without severe hypertension: utility of diffusion-weighted MRI. Neurology. 1998;51(5):1369-76.

24 Calabrese LH, Dodick DW, Schwedt TJ, Singhal AB. Narrative review: reversible cerebral vasoconstriction syndromes. Ann Intern Med. 2007;146(1):34-44.

25 Ducros A. Reversible cerebral vasoconstriction syndrome. Lancet Neurol. 2012;11(10): 906-17. 